\title{
Interferon and Toll-Like Receptor 7 Response in COVID-19: Implications of Topical Imiquimod for Prophylaxis and Treatment
}

\author{
Mindy D. Szeto ${ }^{b} \quad$ Jalal Maghfour ${ }^{a} \quad$ Torunn E. Sivesind $^{b} \quad$ Jarett Anderson ${ }^{c}$ \\ Jadesola T. Olayinkad ${ }^{d}$ Andrina Mamo ${ }^{e}$ Taylor M. Runion $^{f}$ \\ Robert P. Dellavalle ${ }^{b, g}$ \\ aDepartment of Medicine, Tulane University School of Medicine, New Orleans, LA, USA; ${ }^{b}$ Department of \\ Dermatology, University of Colorado Anschutz Medical Campus, Aurora, CO, USA; ' $A$ Arizona College of Osteopathic \\ Medicine, Midwestern University, Glendale, AZ, USA; ${ }^{d}$ College of Medicine, SUNY Downstate Health Sciences \\ Center, New York, NY, USA; ' $S$ Chool of Medicine, University of Colorado Anschutz Medical Campus, Aurora, CO, \\ USA; ${ }^{f}$ College of Osteopathic Medicine, Rocky Vista University, Parker, CO, USA; ${ }^{9}$ Rocky Mountain Regional Veterans \\ Affairs Medical Center, Aurora, CO, USA
}

\section{Keywords}

Imiquimod - Topical imiquimod - Toll-like receptors ·

Interferons · COVID-19

\section{Abstract}

Background: The innate immune system is recognized as an essential aspect of COVID-19 pathogenesis. Toll-like receptors (TLRs) are important in inducing antiviral response, triggering downstream production of interferons (IFNs). Certain loss-of-function variants in TLR7 are associated with increased COVID-19 disease severity, and imiquimod (ImiQ) is known to have immunomodulating effects as an agonist of TLR7. Given that topical imiquimod (toplmiQ) is indicated for various dermatologic conditions, it is necessary for dermatologists to understand the interplay between innate immunity mechanisms and the potential role of ImiQ in COVID-19, with a particular focus on TLR7. Summary: Our objective was to survey recent peer-reviewed scientific literature in the PubMed database, examine relevant evidence, and elucidate the relationships between IFNs, TLR7, the innate im-
\end{abstract}

mune system, and toplmiQ in the context of COVID-19. Despite limited studies on this topic, current evidence supports the critical role of TLRs in mounting a strong immune response against COVID-19. Of particular interest to dermatologists, toplmiQ can result in systemic upregulation of the immune system via activation of TLR7. Key Message: Given the role of TLR7 in the systemic activation of the immune system, ImiQ, as a ligand of the TLR7 receptor, may have potential therapeutic benefit as a topical immunomodulatory treatment for COVID-19.

(c) 2021 S. Karger AG, Basel

\section{Introduction}

COVID-19, caused by Severe Acute Respiratory Syndrome Coronavirus-2 (SARS-CoV-2), was identified as a novel virus (2019-nCoV) in December 2019 and is now

Mindy D. Szeto and Jalal Maghfour share first authorship. 
considered an international public health emergency [1]. A weekly epidemiological report from the World Health Organization (WHO), based on data received on May 9, 2021 [2], reveals that both case and death incidence due to the virus are at maximal levels since the start of the pandemic; worldwide cumulative cases now surpass 157.3 million, with COVID-related deaths totaling over 3.2 million since the pandemic began. According to Centers for Disease Control and Prevention (CDC) data, as of May 5, 2021, there have been over 32 million reported cases and 576,238 deaths related to COVID-19 in the United States alone [3]. It was the third leading cause of daily deaths in the United States in the year 2020, surpassed only by heart disease and cancer [4].

Though COVID-19 is a novel virus, it is a member of the coronaviridae family: viruses characterized by an enveloped membrane and a positive-sense single-stranded RNA (ssRNA) [5]. Knowledge of the immunopathogenesis of COVID-19 primarily builds on previous studies of related coronaviridae, including severe acute respiratory syndrome coronavirus (SARS-CoV) and Middle East Respiratory Syndrome (MERS-CoV). SARS-CoV-2 and SARS-CoV exhibit highly homologous nucleotide sequences, with their genomes differing in only 6 regions [6].

Recent analysis of immunophenotype patterns among hospitalized COVID-19 patients have demonstrated that low levels of type I interferons (IFNs), types of cytokines released by infected cells in response to viruses, which modulate the innate immune response and activate the adaptive immune system, correlate with poor outcomes and prognosis [7]. Additionally, in another study, the administration of IFN- $\alpha 2 b$ (a type I IFN) resulted in reduced viral shedding, as well as lower levels of detectable virus and inflammatory markers in the respiratory tract and blood, respectively [8]. However, the observed variability of IFN response in previous studies suggests that the level and activities of IFN can be dependent upon the host and period of infection. Given its importance in COVID-19 pathogenesis, exploring IFN secretion, responses, and related pathways of the innate immune system could be of clinical utility in elucidating treatments targeting these interrelated mechanisms.

Toll-like receptors (TLRs), important components of the innate immune system, are a family of transmembrane proteins fundamental in activating antiviral signaling pathways such as the above-mentioned type I IFN response. In particular, TLR7 and TLR8 can recognize viral ssRNA. Recent work by Van der Made et al. [9], published in the Journal of the American Medical Association
(JAMA), found that rare loss-of-function variants in the TLR7 gene on the X-chromosome are associated with poor outcomes among hospitalized COVID-19 patients, related to lower than expected levels of type I and type II (which specifically includes IFN- $\gamma$, the main activator of macrophages) IFNs. These findings further support the clinical importance of the innate immune system in COVID-19. Additionally, TLR ligands are currently in development as adjuvants for vaccines against viral and other infectious pathogens [10].

Imiquimod (ImiQ) is a known immunomodulator that exerts biological agonist effects on TLR7 and TLR8, which subsequently activate downstream signaling pathways, leading to increased production of proinflammatory cytokines, such as IFN- $\gamma$, tumor necrosis factor alpha (TNF- $\alpha$ ), and interleukins (IL), including IL- $1 \beta$ and IL-6 [11]. In dermatology, topical imiquimod (topImiQ) is approved for the treatment of actinic keratoses (AK), superficial basal cell carcinoma, and anogenital warts [12]. It is important to recognize that despite being applied topically for dermatologic conditions, ImiQ can also exert systemic effects on the immune system [13]. This review, therefore, aims to discuss in detail the role of IFNs and TLRs in COVID-19 immunopathogenesis and explore the potential clinical significance of ImiQ therapy in relation to COVID-19.

\section{The Link between IFNs, TLR7, and COVID-19 \\ Immunopathogenesis}

IFNs are signaling proteins, grouped into 2 types, that are involved in both the innate and adaptive immune responses; the primary role of type I IFNs, known as the viral IFNs (IFN- $\alpha$, IFN- $\beta$, and secondarily IFN- $\omega$ [14]) is to inhibit viral replication. Type II IFNs include IFN- $\gamma$, predominantly an activator of macrophages [15]. Unlike SARS-CoV and MERS-CoV, strains of SARS-CoV-2 feature either truncations or elongations in the open reading frame ORF3b, which have been suggested to allow SARS$\mathrm{CoV}-2$ to antagonize and inhibit IFN production to a greater extent than SARS-CoV or MERS-CoV [16]. However, SARS-CoV-2 still appears to be highly sensitive to IFN activity. This is illustrated in several clinical studies demonstrating a reduction in viral replication following pre-treatment with type I IFNs in SARS-CoV-2-infected cell lines; this reduction was higher when compared to SARS-CoV-infected cell lines that were pre-treated with type I IFNs [17]. Additionally, in a recent case-control study ( $n=50$ COVID-19 patients; $n=18$ healthy controls), conducted between March 19 and April 3, 2020, low levels of type I IFNs among COVID-19 patients were 
shown to strongly correlate with a poorer clinical prognosis and higher likelihood of transfer to a critical care unit [7]. These results exemplify the importance of IFNs when responding to COVID-19 infection, which has also been established for other viral infections - for example, reduced levels of IFNs have been noted among pediatric patients with greater severity of respiratory syncytial virus infection [18]. However, in contrast to these findings, respiratory syncytial virus and human parainfluenza virus 3 infection was characterized by high IFN and IFNstimulated gene expression [19], and notably, immune hyperactivation can worsen outcomes in COVID-19 [20].

While the exact mechanisms continue to be investigated and better characterized by recent studies, COVID-19 has been confirmed to trigger the activation of innate immune system pathways, the first line of defense against viral pathogens. TLRs play an important role in this immune activation and are comprised of 11 transmembrane proteins expressed on the membranes of many host cells, including leukocytes, epithelial and endothelial cells, and fibroblasts. The most clinically relevant TLRs that have been shown to respond to coronaviruses include TLR4, TLR7/8, and TLR3. TLR4, mainly expressed on a variety of immune system cells, is most noted for its recognition of lipopolysaccharide (LPS) on gram-negative bacteria, but also appears to interact with damage-associated molecular pattern molecules (DAMPs), including oxidized phospholipids released by lung tissue acutely injured by SARS-CoV-2 infection. This interaction results in downstream activation of nuclear factor-kappa B (NF- $\kappa \mathrm{B})$ and induction of type I IFN (including IFN- $\alpha$ and IFN- $\beta$ ) and other proinflammatory cytokine production, such as IL- 6 and TNF- $\alpha$ [21]. While TLR7 and TLR8 both detect ssRNA, with genes in proximity on the X-chromosome, previous research reports that TLR7 is expressed by B cells, monocytes, and plasmacytoid dendritic cells (pDCs). TLR7 agonists also induced a more robust IFN- $\alpha$ and IFN-regulated chemokine response relative to TLR8-selective agonists. Myeloid dendritic cells and monocytes have been observed to generate the greatest TLR8 response (including proinflammatory TNF- $\alpha$ and IL-12 induction) though the cell types expressing TLR8 have yet to be fully characterized [22, 23].

COVID-19 can further activate TLRs through the process of endocytosis. COVID-19 is able to interact with angiotensin converting enzyme-2 receptors, especially those on the type II pneumocytes of the lungs, resulting in endocytosis and transport of viral nucleotides into endosomes. This enables interaction between viral nucleotides, TLR3, and TLR7/8, which are found within the en-

Interferons, TLRs, COVID-19, and

Topical Imiquimod dosomal membrane [24]. Through slightly different pathways, TLR3 and TLR7/8 also activate NF- $\kappa$ B signaling [25], which results in the production of type I IFNs and proinflammatory cytokines IL- 6 , TNF- $\alpha$, and IFN- $\gamma$ [10].

While COVID-19 data continues to be limited, one particular study demonstrated that immune stimulatory activity of SARS-CoV-2 ssRNA was specifically mediated through TLR7 interaction [20]. This interaction likely results in a proinflammatory state necessary to eradicate viruses, but it is important to consider that hyperactivation of the immune system can also contribute to acute lung injury among COVID-19-infected patients [20]. Evidence suggests that hyperactive cytokine release is mediated largely by a few specific cytokines, such as IL-6, a finding further supported by the efficacy of tocilizumab (a monoclonal anti-IL-6 antibody) among patients with severe COVID-19. It has been suggested that an ideal immune response may therefore be facilitated by simultaneously stimulating TLR7 early in the disease course while also inhibiting IL-6 [26].

Recent studies have implicated rare genetic variants in TLR7 as contributors to differential immune response and recovery from COVID-19 infection. In a rapid clinical whole-exome sequencing case series analyzing severe COVID-19 in 4 young, previously healthy male patients (age $<35$ years, two sets of brothers), novel loss-of-function X-chromosomal TLR7 variants were identified, including a 4-nucleotide deletion in the first family and a missense variant in the second family [9]. Notably, these patients did not adequately respond to ImiQ. Four-hour ImiQ stimulation of patients' peripheral blood mononuclear cells resulted in significantly lower than expected increases in TLR7 expression and decreased expression of type I IFN-related genes relative to cells from a healthy control subject. Type II IFN (IFN- $\gamma)$ production was also persistently impaired after 7-day stimulation with ImiQ. These findings suggest a link between the TLR7 loss-offunction variants and defects in IFN response in these patients, potentially contributing to COVID-19 severity and need for hospitalization. Consideration of these TLR7 variants may be especially important in approaching SARS-CoV-2 relative to other coronaviruses. Comparisons of SARS-CoV-2 whole-genome sequencing, relative to SARS-CoV and MERS-CoV, showed that SARSCoV-2 contained a greater number of ssRNA motifs that are able to interact with TLR7 [20].

Additionally, genetic variation in TLR7 may be an underlying factor in observed sex biases in COVID-19 severity, where males could be predisposed to immunode- 


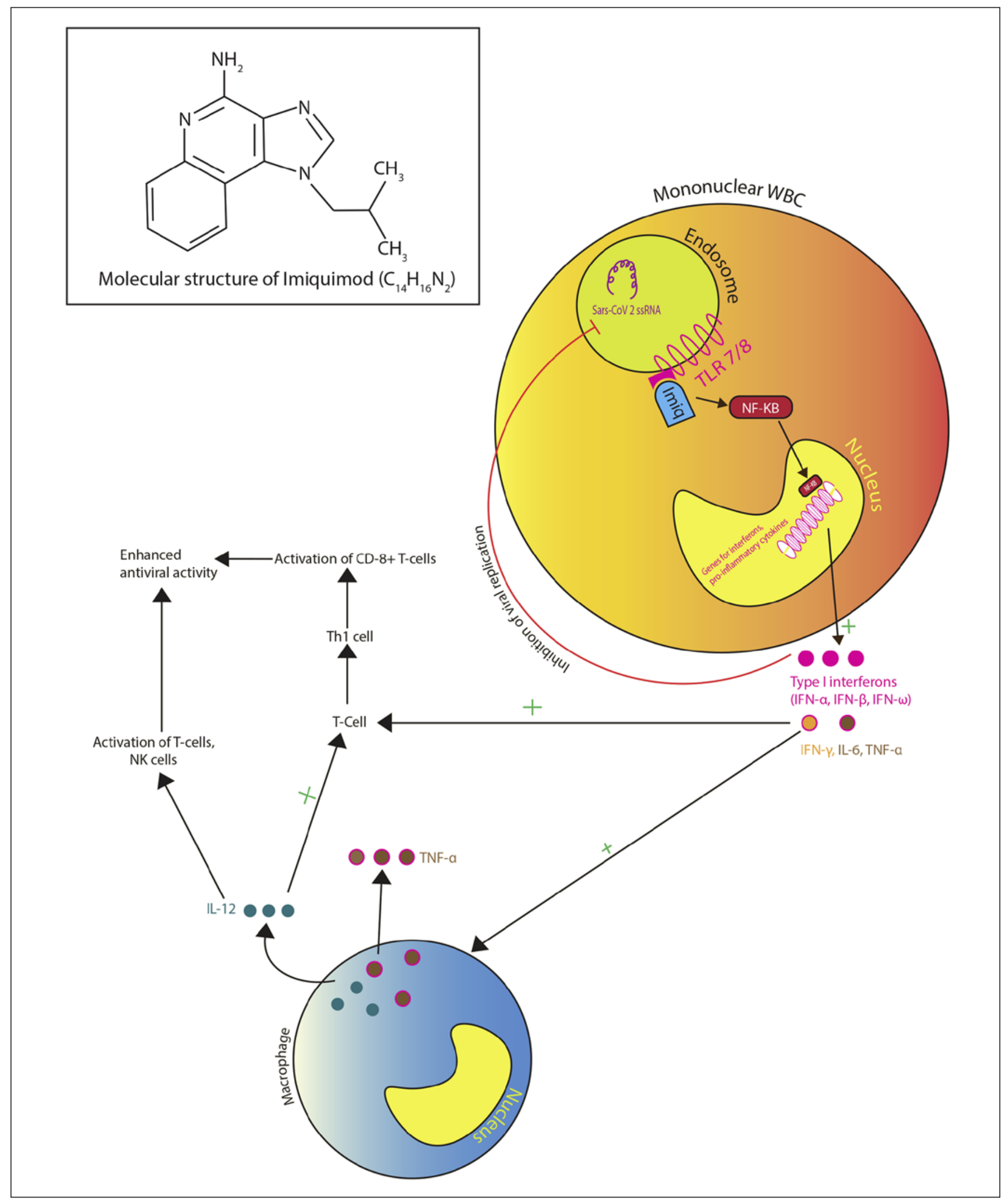

Fig. 1. Structure of imiquimod and overview of related immunological pathways in the context of COVID-19 infection. 
ficient responses due to the location of TLR7 on the Xchromosome [27]. TLR7 also escapes X-inactivation, generally leading to higher basal expression levels and elevated downstream TLR7-induced IFN response in women. Production of IFN- $\alpha$ from pDCs (highly specialized immune cells that can transform into mature dendritic cells and produce type I IFN in response to detected pathogens) is also increased in adult females relative to males, and it has been proposed that higher TLR7 expression among women may facilitate more efficient clearing of viral particles - though perhaps to the detriment of higher autoreactivity and increased risk of autoimmunity [28]. In addition, it was demonstrated that COVID-19 patients had a significantly lower proportion of peripheral pDCs than did healthy controls [7]. Since the process by which pDCs are stimulated to produce IFN is primarily mediated via the TLR7 signaling pathway, therapies that directly stimulate endogenous TLR7, such as ImiQ, could be of clinical importance for the prevention and treatment of COVID-19 infection.

\section{Imiquimod: Mechanism of Action, Antiviral Effects,} and Topical Applications in Dermatology

ImiQ is a potent immunomodulating agent that primarily activates the innate immune system. Its antiviral and antitumor effects have been demonstrated in various animal models [29-31]. ImiQ interacts primarily with TLR7 and TLR8 as a receptor agonist and also promotes TLR7 expression [32]. This results in activation of the NF- $\kappa B$ signaling pathway, which is responsible for the production of proinflammatory cytokines, including IFN- $\alpha$, TNF- $\alpha$, IL-1, IL-6, IL-8, and IL-12, and ultimately promoting an exuberant type 1 helper T-cell (Th1) immune response [12]. A summary of selected pertinent pathways and mechanisms in relation to SARS-CoV-2 and TLR7/8 is shown in Figure 1.

T-cell differentiation into the Th1 subtype is promoted by the production of IL-12 and IFN- $\gamma$. The Th1 effector cytokines include IFN- $\gamma$, IL- 2 , and antitumor TNF- $\beta$, and act to enhance the proliferation of cytotoxic CD8+ T cells, while bolstering the cell-mediated response against intracellular pathogens, including viruses. Additionally, the production of IFN- $\gamma$ activates macrophages - the latter adds to production of IL-12, ultimately resulting in activation of natural killer cells and further activation of Tcells. This mechanism of ImiQ is of clinical importance, as it has been shown that a decline in lymphocyte counts among hospitalized COVID-19 patients is associated with a poor prognosis [33]. Ligands of TLR7, such as ImiQ, have also been shown to induce macrophage pro- duction of inflammatory cytokines IL-23 and IFN- $\beta$ [34]. Expectedly, the aforementioned individuals with TLR7 loss-of-function mutations have demonstrated a failure to mount an appropriate IFN response to ImiQ [9].

Several in vitro, in vivo, and clinical studies have demonstrated the antiviral effects of ImiQ for various other conditions. For instance, ImiQ has been shown to be effective for the treatment of anal condyloma acuminata; the use of suppository ImiQ following surgical ablation resulted in reduced viral loads of associated human papillomavirus (HPV) types 6 and 11 among patients with human immunodeficiency virus (HIV) [35] and, in other studies, was found to prevent recurrence of condylomas [36].

In a study by Grimm et al. [37] among women with high-risk HPV, regression of high-grade cervical intraepithelial neoplasia (CIN) 2-3 was observed following suppository use of ImiQ, with $73 \%$ of those treated achieving CIN 1 or less, and $47 \%$ obtaining total histologic remission; $60 \%$ of those treated also achieved increased HPV viral clearance. The antiviral effects of ImiQ are further supported by a recent study by To et al. [38]: in a murine model, intranasal administration of ImiQ to mice with influenza A virus resulted in reduced viral load and replication, suppressed inflammatory leukocyte infiltration, and decreases in airway inflammation and virally induced lung dysfunction. With the caveat that these studies utilized mucosal application of ImiQ, they do highlight its antiviral effects via modulation of the endogenous immune response.

In dermatology, ImiQ has been well-recognized for its antiviral and antitumor effects, and therefore topImiQ has been used for several decades for the treatment of various dermatologic conditions. Starting in 1997 with an approved indication for external genital and perianal warts (condyloma acuminata), Aldara ${ }^{\circledR} 5 \%$ cream (topImiQ) has received further approval from the US Food and Drug Administration (FDA) for the treatment of AK and superficial basal cell carcinoma. In the context of increasing AK prevalence, and an aging population with a high cumulative exposure to UV radiation, topImiQ holds an important role in field-directed therapy, allowing for treatment of visible AKs while also combating field cancerization [39], an advantage over lesion-directed therapies [40]. Zyclara ${ }^{\circledR}$, a $2.5 \%$ or $3.75 \%$ topImiQ cream, is indicated for daily use over larger skin surface areas than Aldara ${ }^{\circledR}$. Table 1 summarizes topImiQ indications approved by the FDA and the European Medicines Agency (EMA), as well as other known off-label topical applications and potential ImiQ mechanisms of action for viral and neoplastic dermatology-related conditions. 
Table 1. Approved and off-label dermatological indications for topical imiquimod and potential mechanisms of action

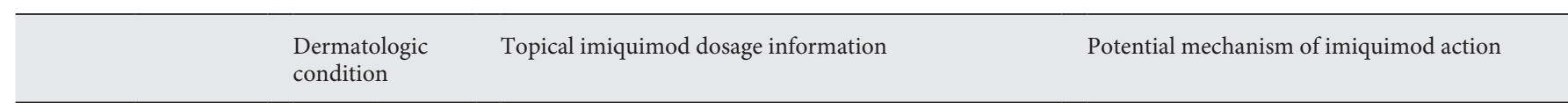

$\begin{array}{lll}\begin{array}{l}\text { Approved indications for } \\ \text { topical imiquimod: }\end{array} & \begin{array}{l}\text { Anogenital warts } \\ \text { (Condyloma }\end{array} & \begin{array}{l}\text { Aldara 5\% Cream (FDA, EMA): } 3 \text { times per week until } \\ \text { total clearance for a maximum of } 16 \text { weeks }[47,48]\end{array}\end{array}$ topical imiquimod: United States Food and Drug Administration (FDA) and European Medicines Agency (EMA) until total clearance for a maximum of 8 weeks [49]

AK Aldara 5\% Cream (FDA): 2 times per week for a full 16 weeks [47] Zyclara $3.75 \%$ Cream or $2.5 \%$ Cream (FDA): Once daily

Aldara 5\% Cream (EMA): 3 times per week for 4 weeks, then assessment of AK clearance over 4-week nontreatment period. Repeat treatment if AK persists and no adverse inflammatory reactions or infections occur [48] Zyclara $3.75 \%$ Cream or $2.5 \%$ Cream (FDA): Once daily to the field area for two 2-week treatment cycles, separated by 2-week non-treatment cycles [49]

Zyclara $3.75 \%$ Cream or $2.5 \%$ Cream (EMA): Once daily to the field area for two 2-week treatment cycles, separated by a 2 -week non-treatment cycle. After 8 week post-treatment evaluation, potential for one more 2 -week treatment if AK persists [51]

Superficial BCC Aldara 5\% Cream (FDA, EMA): 5 times per week for a full 6 weeks $[47,48]$. Not recommended for treatment of BCC subtypes other than the superficial variant [47]

Off-label anti-viral applications of topical imiquimod

Anogenital warts 5\% Suppository: 3 times per week for 16 weeks [35]
(Condyloma
acuminata)
recurrence
prevention

\section{Cervical} intraepithelial neoplasia

Non-genital cutaneous warts

$6.25 \mathrm{mg}$ Vaginal Suppository: 1 per week in weeks 1 and 2, 2 per week in weeks 3 and 4, 3 per week in weeks 5 through 16 [37]

5\% Cream: 5 times a week as tolerated for up to 12 weeks, depending on wart clearance [55]

Herpes simplex $\quad 5 \%$ Cream: For recurrent, chronic, or acyclovir-resistant virus-2 (HSV

herpes genitalis) infection. Results have varied [56-58]

\section{Molluscum} contagiosum

5\% Cream: Prospective, randomized trial in children suggested comparable clearance at 16 weeks with imiquimod (5 days per week) versus cryotherapy ( 1 day per week). Cryotherapy group showed more frequent adverse effects [59]

Off-label anti-neoplastic $\quad$ Bowen's disease $\quad 5 \%$ Cream: Once daily, 5 days per week, for up to 16

applications of topical (squamous cell imiquimod carcinoma in situ) weeks or until clinical clearance [60]. Once daily every other day for 18 weeks showed no residual malignant cells, and no clinical recurrence 6 months post-treatment [61]

Melanoma in situ 5\% Cream: 3-5 times per week for up to 9 months assisted in clearing melanoma in situ with no clinical evidence of recurrence [62]. In another study, once daily application for $5-13$ weeks showed $100 \%$ response rate for lentigo maligna melanoma in situ [63]

Keratoacanthoma 5\% Cream: 3-4 times a week for up to 11 weeks showed almost complete clearance [64]. 4 times a week for 10 weeks eventually resulted in complete resolution with no recurrence [65]. 3-5 weekly applications over 8 weeks induced spontaneous regression [66]
Increased immune response through induction of cytokines IFN- $\alpha$, TNF- $\alpha$, IL-1, IL- 6 , and IL- 8 [50]

Innate and adaptive immune system activation to induce IFN- $\alpha$ and IL-6 [42]. Possible downregulation of anti-apoptotic genes hurpin and HAX-1 [52]. Additional stimulation of vascular E-selectin (not expressed in squamous cell carcinoma) resulting in recruitment of cytotoxic CD8+ T cells with memory T-cell development, limiting progression of subsequent AK [53]

Inhibition of the Hedgehog/glioma-associated oncogene signaling pathway, which is aberrantly activated in BCC. Repressive effect by imiquimod is independent of TLR signaling [54]

Increased immune response through induction of cytokines IFN- $\alpha$, TNF- $\alpha$, IL-1, IL- 6 , and IL- 8 [50]

Stimulates production of IFN, especially IFN- $\alpha$, via TLR7/8 and dendritic cell activation, resulting in T-cell-mediated immune response [37]

Modulation of cell-mediated immunity [55]

No direct antiviral effects. Immunomodulation, via TLR7/8, activates both innate and cellmediated immunity. Subsequent production of IFN- $\gamma$ is effective against HSV infection [56]

Induction of high IFN-a levels localized to the topical application site has potent antiviral properties for treatment of molluscum contagiosum [59]

Activation of innate and adaptive immunity, as well as cytokine stimulation (specifically induction of IFN- $\alpha$ ) has antiviral and antitumor activity, especially given that Bowen's disease is highly associated with HPV 16 and 18 [61]

Activation of innate and adaptive immunity increases lymphocytic infiltration, Langerhans cell (epidermal macrophage) migration, and promotes malignant melanocyte apoptosis $[62,63]$. May also inhibit tumor vascularization [63]

Stimulation of innate and cell-mediated immune response via TLR7/8 activation, including robust IFN-a production, can inhibit rapid squamoproliferative phase and promote spontaneous regression [66]

AK, actinic keratosis; BCC, basal cell carcinoma; HPV, human papillomavirus. 
As there continues to be greater insight regarding the importance of TLR7 in COVID-19 pathogenesis, topImiQ may merit further investigation as a potential prophylactic agent and/or treatment for COVID-19 infection. In a 2020 hypothesis, Avcilar and Eken [26] highlighted the potential benefit of topImiQ for augmentation of the initial immune response to COVID-19 and advocated for further trials exploring its role.

TopImiQ has previously been investigated in combination with intradermal trivalent influenza vaccination. In a double-blind, randomized controlled trial of healthy human volunteers, the use of topImiQ, followed by the intradermal delivery of the influenza vaccine, resulted in seroconversion and improved immunogenicity of nearly all participants in the study group $(n=39 ; 98 \%)$ at day 7 . This was compared to $63 \%(n=25)$ seroconversion in the control group that received influenza vaccination followed only by application of topical aqueous solution. Furthermore, the rate of seroconversion was significantly lower for the control group and for the group receiving topImiQ combined with intradermal saline injection only, compared to the first group that received topImiQ and the vaccine [41].

Consequently, despite its common topical administration as a therapy for various dermatologic conditions (Table 1), there is evidence that topImiQ may result in systemic activation of the immune system. Torres et al. [42] demonstrated statistically significant increased expression of TLR3, TLR7, and TLR8 in peripheral blood mononuclear cells following topImiQ application for the treatment of AK in human male subjects. While increased expression of type I IFN was not directly observed, elevated downstream expression of many IFN-inducible genes was noted, indicating a very quick potential topImiQ response; the authors additionally noted from their prior observations that in human blood mononuclear cells treated with ImiQ, type I IFN mRNA reached maximal levels in 1-2 h, before returning to basal levels $6-8 \mathrm{~h}$ after treatment.

In a murine model of psoriasis, treatment with a 3-day course of 5\% topImiQ resulted in TLR7 activation and increased levels of transcriptional IFN-stimulated genes and upregulation of inflammatory chemokines and cytokines in the skin, peripheral blood leukocytes, liver, and lungs immediately following $4 \mathrm{~h}$ of initial treatment. Anti-inflammatory IL-10 induction was also observed, likely due to regulatory immunosuppression following stimulation of TLR7. Concentration of ImiQ was clearly not limited to the skin, but also reached a significant level in the periphery and the brain, remaining detectable at $12 \mathrm{~h}$,
$24 \mathrm{~h}$, and 3 days after treatment [13]. It should be noted that human epidermis is significantly thicker and contains more cell layers than mouse epidermis; therefore, the penetration of topImiQ and its subsequent systemic effects cannot be directly extrapolated to humans and was a significant limitation of this study's findings.

In a prospective, intention-to-treat study, Kumar and Narang [43] explored systemic and local adverse effects of topImiQ associated with twice-daily dosing (3 days per week for 24 weeks) for the treatment of genital warts in immunocompetent men. Local adverse skin reactions, which are common with topImiQ, were found in $27.3 \%$ of participants. Systemic adverse events, such as fever, fatigue, headache, and myalgia, were found in $36.6 \%$. The occurrence of adverse events was greater than expected, possibly due to increased absorption of ImiQ secondary to local inflammation and the highly vascular nature of the application site. It was also noted that with local induction of cytokines and systemic enhancement of the immune response, the twice-daily dosing regimen used in the study could account for the greater proportion of participants experiencing adverse events.

Harrison et al. [44] studied the pharmacokinetics and safety profile of topImiQ when used in a multi-dosing regimen for the treatment of AK in human adult subjects. Based on analysis of both serum and urine, very low systemic absorption of ImiQ was found, with peak serum accumulation levels of $0.1,0.2$, and $1.6 \mathrm{ng} / \mathrm{mL}$ following the final dosing of face, scalp, and hands/arms application sites, respectively. Minor local application site reactions were the most common adverse events. The authors discussed a known correlation with systemically increased concentration of IFN and other proinflammatory markers after oral administration of ImiQ; however, they postulated that the rise in antiviral inflammatory IFN and also the regulatory anti-inflammatory IL-1 receptor antagonist (IL-1RA) seen following topImiQ administration was the result of spillover from local production of cytokines, rather than systemic induction of the immune response.

Overall, while ImiQ results in activation of various signaling pathways, it primarily modulates the innate immune system by stimulating TLR7. In the past several months, TLR7 has been recognized for playing a critical role in the pathogenesis of COVID-19 - hence, the theoretical value of ImiQ and leveraging the systemic effects of topImiQ as potential treatments for COVID-19 may be inferred. However, it is also worth noting a point of therapeutic divergence in treating COVID-19; for early infections and those with a dampened immune response, it 
may be beneficial to upregulate the immune system via agents such as ImiQ. However, in cases with an exaggerated immune response driving severe respiratory disease, an immunosuppressive agent may instead be warranted [45].

\section{Limitations}

Limitations of our survey include its restriction to a single database (PubMed) and the inclusion of only fulllength English language publications, which may have inadvertently resulted in the omission of certain relevant studies. The rapidly changing nature of the pandemic, along with proliferation of COVID-19 research and related publications, creates a fluctuating landscape of data from which to draw conclusions. Therefore, while we made every effort to utilize the most up-to-date information in writing this review, our findings must be interpreted through the lens of emerging knowledge.

\section{Conclusion}

COVID-19 has created a major global challenge. While COVID-19 vaccine availability and eligibility continue to expand, identifying other avenues for prevention and treatment remain important. This review demonstrated the role of innate immunity, highlighting the functions of TLR7 in immune response and pathogenesis of COVID-19. As a TLR7 agonist, ImiQ has theoretical potential as a possible adjunct therapy to combat COVID-19 infection. Given the increasing use of topImiQ as field treatment for AK among elderly dermatology patients [39], who may be particularly at risk for severe COVID-19 infection due to lower immunity, physicians must be cognizant of the implications of topImiQ with regard to COVID-19 infection.

The upsurge of COVID-19 literature in recent months merits careful consideration of published findings, which will require systematic assessment, verification, and the encouragement of robust investigative studies in large diverse populations. Additional examination of individual genetic variations in TLR7 and other related immunological factors can contribute to a targeted, precision medicine strategy in combating COVID-19.

Finally, bearing in mind previous reports that have demonstrated increased immunogenicity of trivalent influenza vaccines following topImiQ application [41], exploration of a similar approach applied to the new and emerging COVID-19 vaccines is highly necessary. Recently, the emergence of COVID-19 variants, such as
B.1.1.7, B.1.351, and P.1 (also known as the UK, South African, and Brazilian variants, respectively), as well as other variants with multiple mutations, such as B.1.617 [46], has given rise to the concern that the current vaccines may not offer adequate protection against these novel variants. Thus, we hope this provides further impetus for research and future reviews investigating the potential for heightened post-vaccine seroconversion secondary to ImiQ.

\section{Key Message}

Topical Toll-like receptor agonist imiquimod can stimulate systemic innate immunity, pointing to implications for COVID-19 immunopathogenesis and treatment.

\section{Conflict of Interest Statement}

Dr. Dellavalle is Editor in Chief of JMIR Dermatology, a Joint Coordinating Editor for Cochrane Skin, a Dermatology Section Editor for UpToDate, a Social Media Editor for the Journal of the American Academy of Dermatology (JAAD), and a Podcast Editor for the Journal of Investigative Dermatology (JID). He is a Coordinating Editor representative on Cochrane Council. Dr. Sivesind is a Section Editor for JMIR Dermatology.

\section{Funding Sources}

Dr. Dellavalle receives editorial stipends (JAAD, JID, JMIR Dermatology), royalties (UpToDate), and expense reimbursement from Cochrane Skin. Dr. Sivesind receives fellowship funding from the Pfizer Global Medical Grant (58858477) Dermatology Fellowship 2020 (PI: R.P. Dellavalle), and receives fees for serving as a Medical Advisor and Investigator for Antedotum Inc.

\section{Author Contributions}

M.D.S., J.M., and T.E.S. provided substantial contributions to the conception and design of the work, drafting of the work, and critical revision. J.A. contributed to figure creation and assisted in drafting of the work. J.T.O. contributed to table creation and assisted in drafting of the work. A.M. and T.M.R. assisted in drafting and revision of the work. R.P.D. guided the literature search and design of the work and contributed to critical revision. All authors provided final approval and agreed to be accountable for all aspects of the work in ensuring that questions related to the accuracy or integrity of any part of the work are appropriately investigated and resolved. 


\section{References}

1 Zhu N, Zhang D, Wang W, Li X, Yang B, Song J, et al. A Novel Coronavirus from Patients with Pneumonia in China, 2019. N Engl J Med. 2020 Feb;382(8):727-33.

2 World Health Organization. Weekly Epidemiological Update. [cited May 16, 2021]. Available from: https://www.who.int/ publications/m/item/weekly-epidemiological-update-on-covid-19---11-may-2021.

3 Centers for Disease Control and Prevention. Covid Data Tracker Weekly Review. [cited May 16, 2021]. Available from: https://www. cdc.gov/coronavirus/2019-ncov/covid-data/ covidview/index.html.

4 Centers for Disease Control and Prevention. Morbidity and Mortality Weekly Report (MMWR): Provisional Mortality Data United States, 2020. [cited May 16, 2021]. Available from: https://www.cdc.gov/mmwr/ volumes/70/wr/mm7014e1.htm.

5 Li G, Fan Y, Lai Y, Han T, Li Z, Zhou P, et al. Coronavirus infections and immune responses. J Med Virol. 2020 Apr;92(4):424-32.

6 Kaur N, Singh R, Dar Z, Bijarnia RK, Dhingra N, Kaur T. Genetic comparison among various coronavirus strains for the identification of potential vaccine targets of SARS-CoV2. Infect Genet Evol. 2021;89:104490.

7 Hadjadj J, Yatim N, Barnabei L, Corneau A, Boussier J, Smith N, et al. Impaired type I interferon activity and inflammatory responses in severe COVID-19 patients. Science. 2020 Aug;369(6504):718-24.

8 Zhou Q, Chen V, Shannon CP, Wei XS, Xiang $\mathrm{X}$, Wang $\mathrm{X}$, et al. Interferon- $\alpha 2 \mathrm{~b}$ Treatment for COVID-19. Front Immunol. 2020;11: 1061.

9 van der Made CI, Simons A, Schuurs-Hoeijmakers J, van den Heuvel G, Mantere T, Kersten S, et al. Presence of Genetic Variants among Young Men with Severe COVID-19. JAMA. 2020;324(7):663-73.

10 Kawai T, Akira S. Toll-like receptors and their crosstalk with other innate receptors in infection and immunity. Immunity. 2011 May; 34(5):637-50.

11 Angelopoulou A, Alexandris N, Konstantinou E, Mesiakaris K, Zanidis C, Farsalinos K, et al. Imiquimod - a toll like receptor 7 agonist - is an ideal option for management of COVID 19. Environ Res. 2020 Sep;188:109858.

12 Hanna E, Abadi R, Abbas O. Imiquimod in dermatology: an overview. Int J Dermatol. 2016 Aug;55(8):831-44.

13 Nerurkar L, McColl A, Graham G, Cavanagh J. The systemic response to topical aldara treatment is mediated through direct TLR7 stimulation as imiquimod enters the circulation. Sci Rep. 2017 Nov;7(1):16570.

14 Seo Y, Kim M, Choi M, Kim S, Park K, Oh I, et al. Possible role of phosphoinositide-3-kinase in $\mathrm{Mx} 1$ protein translation and antiviral activity of interferon-omega-stimulated HeLa cells. Pharmacology. 2011;87(3-4):224-31.
15 Samuel CE. Antiviral actions of interferons. Clin Microbiol Rev. 2001 Oct;14(4):778-809.

16 Konno Y, Kimura I, Uriu K, Fukushi M, Irie T, Koyanagi Y, et al. SARS-CoV-2 ORF3b Is a Potent Interferon Antagonist Whose Activity Is Increased by a Naturally Occurring Elongation Variant. Cell Rep. 2020 Sep;32(12): 108185.

17 Lokugamage KG, Hage A, de Vries M, Valero-Jimenez AM, Schindewolf C, Dittmann M, et al. Type I Interferon Susceptibility Distinguishes SARS-CoV-2 from SARS-CoV. J Virol. 2020 Nov;94(23):e01410-20.

18 Heinonen S, Velazquez VM, Ye F, Mertz S, Acero-Bedoya S, Smith B, et al. Immune profiles provide insights into respiratory syncytial virus disease severity in young children. Sci Transl Med. 2020 Apr;12(540):eaaw0268.

19 Blanco-Melo D, Nilsson-Payant BE, Liu WC, Uhl S, Hoagland D, Møller R, et al. Imbalanced Host Response to SARS-CoV-2 Drives Development of COVID-19. Cell. 2020 May; 181(5):1036-1045.e9.

20 Moreno-Eutimio MA, López-Macías C, Pastelin-Palacios R. Bioinformatic analysis and identification of single-stranded RNA sequences recognized by TLR7/8 in the SARSCoV-2, SARS-CoV, and MERS-CoV genomes. Microbes Infect. 2020 May-Jun;22(45):226-9.

21 Imai Y, Kuba K, Neely GG, Yaghubian-Malhami R, Perkmann T, van Loo G, et al. Identification of oxidative stress and Toll-like receptor 4 signaling as a key pathway of acute lung injury. Cell. 2008;133(2):235-49.

22 Gorden KB, Gorski KS, Gibson SJ, Kedl RM, Kieper WC, Qiu X, et al. Synthetic TLR agonists reveal functional differences between human TLR7 and TLR8. J Immunol. 2005 Feb;174(3):1259-68.

23 Bender AT, Tzvetkov E, Pereira A, Wu Y, Kasar S, Przetak MM, et al. TLR7 and TLR8 Differentially Activate the IRF and NF- $\kappa$ B Pathways in Specific Cell Types to Promote Inflammation. Immunohorizons. $2020 \mathrm{Feb}$; 4(2):93-107.

24 Ni W, Yang X, Yang D, Bao J, Li R, Xiao Y, et al. Role of angiotensin-converting enzyme 2 (ACE2) in COVID-19. Crit Care. 2020 Jul 13; 24(1):422.

25 Kawai T, Akira S. Signaling to NF-kappaB by Toll-like receptors. Trends Mol Med. 2007 Nov;13(11):460-9.

26 Avcilar H, Eken A. Could imiquimod (Aldara $5 \%$ cream) or other TLR7 agonists be used in the treatment of COVID-19?. Med Hypotheses. 2020 Nov; 144:110202.

27 Scully EP, Haverfield J, Ursin RL, Tannenbaum C, Klein SL. Considering how biological sex impacts immune responses and COVID-19 outcomes. Nat Rev Immunol. 2020; 20(7):442-7.
28 Souyris M, Mejía JE, Chaumeil J, Guéry JC. Female predisposition to TLR7-driven autoimmunity: gene dosage and the escape from $\mathrm{X}$ chromosome inactivation. Semin Immunopathol. 2019 Mar;41(2):153-64.

29 Reiter MJ, Testerman TL, Miller RL, Weeks CE, Tomai MA. Cytokine induction in mice by the immunomodulator imiquimod. J Leukoc Biol. 1994 Feb;55(2):234-40.

30 Dahl MV. Imiquimod: a cytokine inducer. J Am Acad Dermatol. 2002 Oct;47(4 Suppl 1): S205-8.

31 Gupta AK, Browne M, Bluhm R. Imiquimod: a review. J Cutan Med Surg. 2002 Nov-Dec; 6(6):554-60.

32 Li ZJ, Sohn KC, Choi DK, Shi G, Hong D, Lee $\mathrm{HE}$, et al. Roles of TLR7 in activation of NF$\kappa \mathrm{B}$ signaling of keratinocytes by imiquimod. PLoS One. 2013;8(10):e77159.

33 Xu B, Fan CY, Wang AL, Zou YL, Yu YH, He C, et al. Suppressed T cell-mediated immunity in patients with COVID-19: A clinical retrospective study in Wuhan, China. J Infect. 2020 Jul;81(1):e51-60.

34 Al-Salleeh F, Petro TM. TLR3 and TLR7 are involved in expression of IL-23 subunits while TLR3 but not TLR7 is involved in expression of IFN-beta by Theiler's virus-infected RAW264.7 cells. Microbes Infect. 2007 Sep; 9(11):1384-92.

35 Kreuter A, Brockmeyer NH, Weissenborn SJ, Wafaisade A, Pfister H, Altmeyer P, et al. 5\% Imiquimod suppositories decrease the DNA load of intra-anal HPV types 6 and 11 in HIVinfected men after surgical ablation of condylomata acuminata. Arch Dermatol. 2006; 142(2):243-4.

36 Kaspari M, Gutzmer R, Kaspari T, Kapp A, Brodersen JP. Application of imiquimod by suppositories (anal tampons) efficiently prevents recurrences after ablation of anal canal condyloma. Br J Dermatol. 2002 Oct;147(4): 757-9.

37 Grimm C, Polterauer S, Natter C, Rahhal J, Hefler L, Tempfer CB, et al. Treatment of cervical intraepithelial neoplasia with topical imiquimod: a randomized controlled trial. Obstet Gynecol. 2012 Jul;120(1):152-9.

38 To EE, Erlich J, Liong F, Luong R, Liong S, Bozinovski S, et al. Intranasal and epicutaneous administration of Toll-like receptor 7 (TLR7) agonists provides protection against influenza A virus-induced morbidity in mice. Sci Rep. 2019 Feb;9(1):2366.

39 Stockfleth E. The importance of treating the field in actinic keratosis. J Eur Acad Dermatol Venereol. 2017 Mar;31(Suppl 2):8-11.

40 Piquero-Casals J, Morgado-Carrasco D, Gilaberte Y, Del Rio R, Macaya-Pascual A, Granger $\mathrm{C}$, et al. Management pearls on the treatment of actinic keratoses and field cancerization. Dermatol Ther (Heidelb). 2020 Oct; 10(5):903-15.
Interferons, TLRs, COVID-19, and Topical Imiquimod
Dermatology 2021;237:847-856

DOI: $10.1159 / 000518471$ 
41 Hung IF, Zhang AJ, To KK, Chan JF, Li P, Wong TL, et al. Topical imiquimod before intradermal trivalent influenza vaccine for protection against heterologous non-vaccine and antigenically drifted viruses: a single-centre, double-blind, randomised, controlled phase 2b/3 trial. Lancet Infect Dis. 2016 Feb;16(2): 209-18.

42 Torres A, Storey L, Anders M, Miller RL, Bulbulian BJ, Jin J, et al. Immune-mediated changes in actinic keratosis following topical treatment with imiquimod $5 \%$ cream. J Transl Med. 2007 Jan;5:7.

43 Kumar B, Narang T. Local and systemic adverse effects to topical imiquimod due to systemic immune stimulation. Sex Transm Infect. 2011 Aug;87(5):432.

44 Harrison LI, Skinner SL, Marbury TC, Owens ML, Kurup S, McKane S, et al. Pharmacokinetics and safety of imiquimod 5\% cream in the treatment of actinic keratoses of the face, scalp, or hands and arms. Arch Dermatol Res. 2004 Jun;296(1):6-11.

45 Plenge RM. Molecular Underpinnings of Severe Coronavirus Disease 2019. JAMA. 2020; 324(7):638-9.

46 Centers for Disease Control and Prevention. Science Brief: Emerging SARS-CoV-2 Variants. Updated Jan 28, 2021 [cited May 16, 2021]. Available from: https://www.cdc.gov/ coronavirus/2019-ncov/science/sciencebriefs/scientific-brief-emerging-variants.html.

47 Aldara $^{\star}$ (imiquimod) Cream, 5\% [package insert]. United States Food and Drug Administration. Revised October 2010 [cited May 16, 2021]. Available from: https://www.accessdata.fda.gov/drugsatfda_docs/label/2010/ 020723s022lbl.pdf.

48 Aldara 5\% cream. European Medicines Agency: European Public Assessment Report Product Information. Revised May 6, 2021 [cited May 16, 2021]. Available from: https:// www.ema.europa.eu/en/documents/product-information/aldara-epar-product-information_en.pdf.

49 Zyclara $^{\circ}$ (imiquimod) cream, 3.75\% and 2.5\% [package insert]. United States Food and
Drug Administration. Revised July 2011 [cited May 16, 2021]. Available from: https:// www.accessdata.fda.gov/drugsatfda_docs/ label/2011/022483s003lbl.pdf.

50 Arany I, Tyring SK, Brysk MM, Stanley MA, Tomai MA, Miller RL, et al. Correlation between pretreatment levels of interferon response genes and clinical responses to an immune response modifier (Imiquimod) in genital warts. Antimicrob Agents Chemother. 2000 Jul;44(7):1869-73.

51 Zyclara 3.75\% cream. European Medicines Agency: European Public Assessment Report - Product Information. Revised Jan 24, 2018 [cited May 16, 2021]. Available from: https:// www.ema.europa.eu/en/documents/product-information/zyclara-epar-product-information_en.pdf.

52 Lysa B, Tartler U, Wolf R, Arenberger P, Benninghoff $B$, Ruzicka $T$, et al. Gene expression in actinic keratoses: pharmacological modulation by imiquimod. Br J Dermatol. 2004 Dec;151(6):1150-9.

53 Clark RA, Huang SJ, Murphy GF, Mollet IG, Hijnen D, Muthukuru M, et al. Human squamous cell carcinomas evade the immune response by down-regulation of vascular E-selectin and recruitment of regulatory $\mathrm{T}$ cells. J Exp Med. 2008 Sep;205(10):2221-34.

54 Wolff F, Loipetzberger A, Gruber W, Esterbauer H, Aberger F, Frischauf AM. Imiquimod directly inhibits Hedgehog signalling by stimulating adenosine receptor/protein kinase A-mediated GLI phosphorylation. Oncogene. 2013 Dec;32(50):5574-81.

55 Hengge UR, Esser S, Schultewolter T, Behrendt C, Meyer T, Stockfleth E, et al. Self-administered topical 5\% imiquimod for the treatment of common warts and molluscum contagiosum. Br J Dermatol. 2000 Nov; 143(5):1026-31.

56 Gilbert J, Drehs MM, Weinberg JM. Topical Imiquimod for Acyclovir-Unresponsive Herpes Simplex Virus 2 Infection. Arch Dermatol. 2001;137(8):1015-7.

57 Bernstein DI, Spruance SL, Arora SS, Schroeder JL, Meng TC. Evaluation of Imi- quimod 5\% Cream to Modify the Natural History of Herpes Labialis: A Pilot Study. Clin Infect Dis. 2005;41(6):808-14.

58 Bangsgaard N, Skov L. Chronic genital ulceration due to herpes simplex infection treated successfully with imiquimod. Acta Derm Venereol. 2008;88(2):202-3.

59 Al-Mutairi N, Al-Doukhi A, Al-Farag S, AlHaddad A. Comparative study on the efficacy, safety, and acceptability of imiquimod 5\% cream versus cryotherapy for molluscum contagiosum in children. Pediatr Dermatol. 2010 Jul-Aug;27(4):388-94.

60 Peris K, Micantonio T, Fargnoli MC, Lozzi GP, Chimenti S. Imiquimod 5\% cream in the treatment of Bowen's disease and invasive squamous cell carcinoma. J Am Acad Dermatol. 2006 Aug;55(2):324-7.

61 Yanagishita T, Akita Y, Nakanishi G, Tamada Y, Watanabe D. Pigmented Bowen's disease of the digit successfully treated with imiquimod 5\% cream. Eur J Dermatol. 2011 NovDec;21(6):1021-2.

62 Fan Q, Cohen S, John B, Riker AI. Melanoma in Situ Treated with Topical Imiquimod for Management of Persistently Positive Margins: A Review of Treatment Methods. Ochsner J. 2015 Winter;15(4):443-7.

63 Wolf IH, Cerroni L, Kodama K, Kerl H. Treatment of Lentigo Maligna (Melanoma in situ) with the Immune Response Modifier Imiquimod. Arch Dermatol. 2005;141(4):510-4.

64 Jeon HC, Choi M, Paik SH, Ahn CH, Park HS, Cho KH. Treatment of keratoacanthoma with $5 \%$ imiquimod cream and review of the previous report. Ann Dermatol. 2011 Aug;23(3): 357-61.

65 Pancevski G, Pepic S, Idoska S, Tofoski G, Nikolovska S. Topical Imiquimod $5 \%$ as a Treatment Option in Solitary Facial Keratoacanthoma. Open Access Maced J Med Sci. 2018 Mar;6(3):531-5.

66 Di Lernia V, Ricci C, Albertini G. Spontaneous regression of keratoacanthoma can be promoted by topical treatment with imiquimod cream. J Eur Acad Dermatol Venereol. 2004 Sep;18(5):626-9. 